\title{
Prevention of central venous catheter-related infection in the intensive care unit
}

\author{
Denis Frasca, Claire Dahyot-Fizelier, Olivier Mimoz* \\ This article is one of ten reviews selected from the Yearbook of Intensive Care and Emergency Medicine 2010 (Springer Verlag) and co-published \\ as a series in Critical Care. Other articles in the series can be found online at http://ccforum/series/yearbook. Further information about the \\ Yearbook of Intensive Care and Emergency Medicine is available from http://www.springer.com/series/2855.
}

\section{Introduction}

In the USA, more than five million patients require central venous access each year. Unfortunately, central venous access can be associated with adverse events that are hazardous to patients and expensive to treat. Infection remains the main complication of intravascular catheters in critically ill patients. Catheter-related bloodstream infections have been reported to occur in 3 to $8 \%$ of inserted catheters and are the first cause of nosocomial bloodstream infection in intensive care units (ICUs), with 80,000 cases annually at a cost of $\$ 300$ million to $\$ 2.3$ billon [1]. Additional financial costs may be as high as $\$ 30,000$ per survivor, including one extra week in the ICU and two to three additional weeks in the hospital. Attributable mortality rates range from 0 to $35 \%$, depending on the degree of control for severity of illness.

The physiopathology of catheter infection is now more clearly understood. Colonization of the endovascular tip of the catheter precedes infection and arises by two main pathways: The extraluminal and the intraluminal routes (Fig. 1) [2]. Migration of skin organisms from the insertion site into the cutaneous catheter tract with colonization of the catheter tip is the most common route of infection for short-term central venous catheters (CVCs). For long-term catheters (i.e., catheters staying in place more than 15 days), the main cause of colonization is manipulation of the venous line with migration of organisms along the internal lumen of the catheter. The adherence properties of microorganisms to host proteins, such as fibronectin, commonly present on catheter tips make this colonization easier. Coagulase-negative staphylococci are the most common microorganisms

*Correspondence: o.mimoz@chu-poitiers.fr

Surgical Intensive Care, Centre Hospitalier Universitaire, 2 rue de la Mileterie, 86021 Poitiers, France associated with catheter-related bloodstream infections. Other microorganisms commonly involved include Staphylococcus aureus, Candida species, Enterococci and Gram-negative bacilli [3].

The Centers for Disease Control and Prevention identifies catheter-associated adverse events, including bloodstream infections, as one of its seven health care safety challenges, with a goal to reduce such complications by $50 \%$ in five years [4]. Several preventive measures have been studied to reduce the incidence of these infections. The most effective are those that reduce colonization at the catheter skin insertion site or the infusion line, and include: Adequate knowledge and use of care protocols; qualified personnel involved in catheter changing and care; use of biomaterials that inhibit microorganism growth and adhesion; good hand hygiene; use of an alcoholic formulation of chlorhexidine for skin disinfection and manipulation of the vascular line; preference for the subclavian vein route for insertion of CVCs using full-barrier precautions; and removal of unnecessary catheters.

\section{Catheter care protocols}

Programs that help health-care providers to monitor and evaluate care are crucial for the success of preventive measures. Educational programs with hygiene training and written protocols concerning catheter insertion (e.g., preparation of the equipment, skin antisepsis, detailed insertion techniques), catheter manipulation (e.g., hand hygiene, manipulations of taps) and catheter care (e.g., catheter replacement modalities, type and frequency of dressings, and line repair) are effective when staff members are involved in designing the measures included in the program $[5,6]$. Regular evaluation of the incidence of catheter-related infections and of clinical practice is a useful measure when information and 


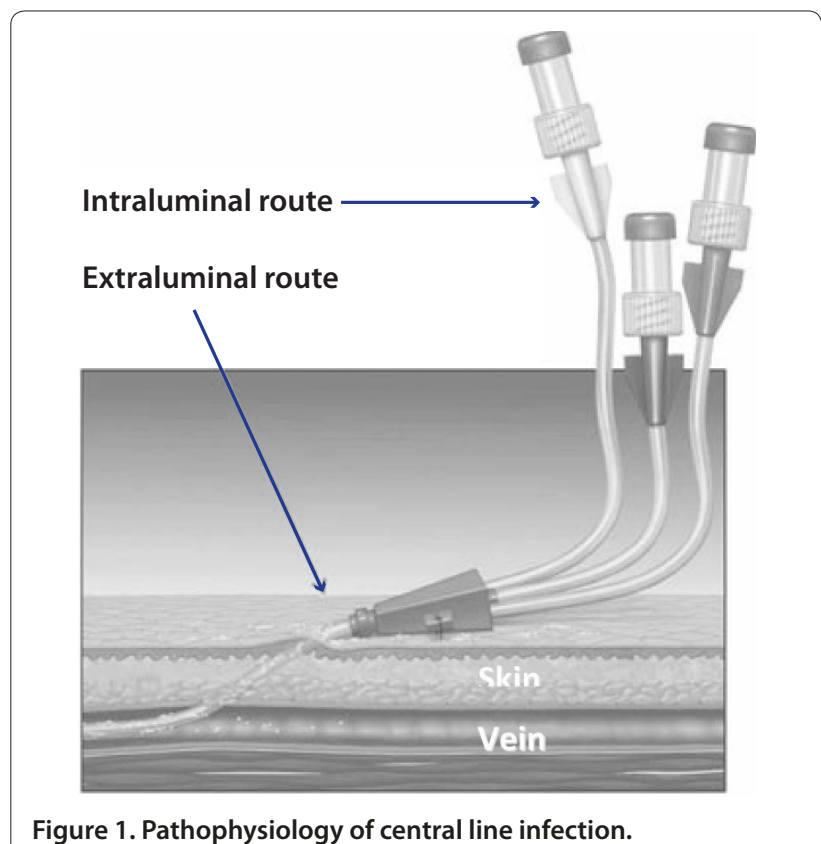

feedback is provided to all actors $[7,8]$. Catheter insertion in emergency conditions increases the risk of non-compliance to the insertion protocol and, consequently, to infectious complications; these catheters must be replaced as soon as the patient's condition is stabilized [9].

\section{Staff educational/quality improvement program}

Educating and training of health-care providers who insert and maintain CVCs is essential for preventing catheter-related infection, improving patient outcomes, and reducing healthcare costs [10]. The experience of the operator is an important issue as the risk of infectious complications is inversely proportional to the operator skills. An educational intervention in catheter insertion significantly improved patient outcomes and simulationbased training programs are valuable in residency education [11]. Programs for training nurses in longterm catheter care ("IV teams") were associated with a reduction in catheter-related infections in the USA [12]. Nevertheless, without such teams the use of care protocols and nursing staff education allowed comparable results to be obtained [13]. Nursing staff reductions below a critical level may contribute to increase catheterrelated infection by making adequate catheter care difficult. One study reported a four times greater risk of catheter infection when the patient-to-nurse ratio was doubled [14]. Moreover, replacement of regular nurses by float nurses further increases the risk of device-related infections [15]. These studies clearly indicate that trained nurses, in sufficient numbers, must be available for optimal patient care in the ICU.

\section{Type of catheter}

Catheter material is an important determinant in the prevention of catheter-related infection. The material should be biocompatible, hemocompatible, biostable, chemically neutral, not altered by administered drugs, and deformable according to surrounding strengths. Furthermore, the catheter must be flexible, resistant, as radio-opaque as possible, thin walled with a high internal to external diameter ratio, resistant to sterilization, and with locked connections such as 'luer-lock' type. Teflon ${ }^{\circ}$ or polyurethane catheters have been associated with fewer infectious complications than catheters made of polyvinyl chloride or polyethylene $[16,17]$. The majority of catheters sold in the USA and in many European countries are, therefore, no longer made of polyvinyl chloride or polyethylene.

Catheters coated with antimicrobial or antiseptic agents decrease microorganism adhesion and biofilm production, and, hence, the risk of catheter-related infection. The use of such catheters may potentially decrease hospital costs, despite the additional acquisition cost of the antimicrobial/antiseptic coated catheter [18]. Commercialized catheters are mainly coated with chlorhexidine/ silver sulfadiazine or minocycline/rifampin [19]. Fifteen randomized studies evaluating the performance of a catheter coated on its extraluminal side with chlorhexidine/silver sulfadiazine (first generation) were included in a meta-analysis. Compared to a standard catheter, the use of the coated catheter decreased the risk of catheter colonization (relative risk, RR: 0.59 [95\% CI: 0.50-0.71]) and bloodstream infection (RR: 0.66 [95\% CI: 0.47-0.93]) [20]. Two studies evaluated catheters coated on both their external and internal surfaces (second generation) and provided comparable results concerning colonization (RR: 0.44 [95\% CI: 0.23-0.85]) and a non-significant reduction in bloodstream infection (RR: 0.70 [95\% CI: $0.30-1.62]$ ), probably due to a lack of power. Five studies evaluated catheters covered with minocycline/rifampin and reported a decrease in colonization (RR: 0.40 [95\% CI: 0.23-0.67]) and bloodstream infection (RR: 0.39 [95\% CI: 0.17-0.92]) compared to standard catheters. Two studies concluded that silver-coated catheters (even with platinum or carbon coating) had no beneficial effects on colonization (RR: 0.76 [95\% CI: 0.57-1.01]) or on bloodstream infection (RR: 0.54 [95\% CI: 0.16-1.85]), but the studies were underpowered. A multicenter randomized study evaluated catheters impregnated with ionic silver in 577 ICU patients and 617 CVCs [21]. Compared to standard catheters, impregnated catheters had no effect on colonization (RR: 1.24 [95\% CI: 0.83-1.85]) or bloodstream infection prevention (RR: 0.93 [95\% CI: 0.35-2.44]). Two studies compared first generation antiseptic catheters with antibiotic-coated catheters and concluded that the latter were superior for preventing 
catheter colonization (RR: 0.36 [95\% CI: $0.25-0.53]$ ) and bloodstream infection (RR: 0.12 [95\% CI: 0.02-0.67]). No study has compared antibiotic-coated catheters with second generation antiseptic impregnated catheters. At this time, there is no evidence for multi-resistant bacteria selection with antibiotic-coated catheters, but the number of studies is limited. Rare but serious cases of anaphylactic reactions to chlorhexidine/silver sulfadiazine have been reported, mainly in Japan. However, despite a Food and Drug Administration (FDA) alert in 1998 encouraging the declaration of these events, the number of cases reported in the USA remains low. Considering their costs and their theoretical ecological impact, the use of CVCs coated with antimicrobial agents should be reserved for ICUs where the incidence of catheter-related infection remains high despite adherence to guidelines and recommended measures [22].

CVCs with multiple lumens allow simultaneous administration of incompatible drugs and may separate the administration of vasopressors and parenteral nutrition. Five randomized studies have evaluated the risk of the use of multilumen catheters on catheter colonization and bloodstream infection [23]. Most of these studies are old, were conducted outside the ICU, and included few patients. Compared to mono-lumen catheters, the use of multiple lumen catheters was associated with comparable risks of catheter colonization (RR: 0.80 [95\% CI: 0.43-1.50]), but higher risks of bloodstream infection (RR: 2.26 [95\% CI: 1.06-4.83]). The increased risk of bloodstream infection is explained by one study which included long-term catheters (mean duration of catheterization longer than 20 days) for parenteral nutrition and reported a surprisingly high level of infection with multiple lumen catheters (13.1\% versus $2.6 \%$ with mono-lumen catheters). Excluding this study from the meta-analysis gave a comparable risk of bloodstream infection between the groups (RR: 1.29 [95\% CI: 0.49-3.39]). The choice of the number of lumens should, therefore, be made based on the patient's requirements rather than on the risk of infectious complications. Any solution containing lipids (parenteral nutrition, propofol) must be delivered through a dedicated lumen.

\section{Catheter insertion site}

The site at which a catheter is inserted may influence the subsequent risk of catheter-related infection because of differences in the density of local skin flora and risks of thrombophlebitis. A randomized study of 270 catheters inserted in the femoral or subclavian veins of ICU patients [24] reported a higher colonization rate with femoral catheters (RR: 6.4 [ 95\% CI: 1.9-21.2]) without any increase in bloodstream infections (RR: 2.0 [ 95\% CI: $0.2-22.1]$ ). A meta-analysis of three prospective nonrandomized studies compared catheters inserted in the internal jugular $(\mathrm{n}=278)$ and subclavian $(\mathrm{n}=429)$ veins. The use of the internal jugular vein was associated with a non-significant increase in the risk of bloodstream infection (RR: 2.24 [95\% CI: 0.2-22.1]) compared to the subclavian route. Moreover, multivariate analysis of several prospective studies has shown more frequent infectious complications when using femoral or internal jugular access [25].

A randomized multicenter study evaluated the risk of complications with dialysis catheters in the ICU according to femoral or internal jugular insertion site. A total of 750 catheters with an average duration of insertion of 6 days were included. The risk for colonization was comparable for both sites (incidence of 40.8 vs 35.7 per 1000 catheter-days for the femoral and jugular sites, respectively, RR: 0.85 [95\% CI: 0.62-1.16]). Nevertheless, the risk of colonization with internal jugular access was increased in patients with a body mass index less than 24.2 (RR: 2.10 [95\% CI: 0.23-0.69]) and decreased in patients with a body mass index greater than 28.4 (RR: 0.40 [95\% CI: 1.13-3.91]) [26].

The subclavian site is preferred for infection control purposes, although other factors (e.g., the potential for mechanical complications, risk of subclavian vein stenosis, and catheter-operator skill) should be considered when deciding where to place the catheter. When the subclavian route is contraindicated, the choice between the femoral and internal jugular vein should be made according to the body mass index of the patient. The risk of thrombophlebitis should also be taken into consideration, as it is higher with the femoral route than when using the subclavian or internal jugular veins.

\section{Ultrasound-guided placement}

The use of ultrasound guidance has been promoted as a method to reduce the risk of complications during central venous catheterization. In this technique, an ultrasound probe is used to localize the vein and to measure its depth beneath the skin. Under ultrasound visualization, the introducer needle is then guided through the skin and into the vessel. The location of the vein with ultrasound decreases the number of puncture failures and complications (e.g., arterial puncture), and reduces the time for catheter insertion. This technique may provide advantages for the jugular internal vein location. In a meta-analysis of eight studies, the use of bedside ultrasound for the placement of catheters substantially reduced mechanical complications compared with the standard landmark placement technique (RR: 0.22; [95\% CI: 0.10-0.45]) [27]. Data available for subclavian or femoral veins are encouraging but limited. In a randomized study with 900 ICU patients, ultrasound-guided placement resulted in a reduction in bloodstream infection $(10.4 \%$ vs $16.0 \%, p<0.01)$ [28]. In hospitals where 
ultrasound equipment is available and physicians have adequate training, the use of ultrasound guidance should be routinely considered before $\mathrm{CVC}$ placement is attempted.

\section{Insertion technique}

When inserting a catheter, one should use maximal sterile-barrier precautions, including a mask, a cap, a sterile gown, sterile gloves, and a large sterile drape. This approach has been shown to reduce the rate of catheterrelated bloodstream infections and to save an estimated $\$ 167$ per catheter inserted [29]. The insertion site should be widely disinfected with a chlorhexidine-based solution. Catheters should then be inserted using the Seldinger technique and adequately secured.

\section{Skin antisepsis}

The density of microorganisms at the catheter insertion site is a major risk factor for catheter-related infection and skin antisepsis is one of the most important preventive measures. Povidone iodine and chlorhexidine are the most commonly used antiseptic agents, both available as aqueous and alcoholic solutions. Their respective efficacy in preventing catheter colonization and bloodstream infections has been compared in numerous studies.

One meta-analysis included eight randomized trials that compared chlorhexidine to aqueous povidone iodine for the care of 4143 short-term catheters $(1568 \mathrm{CVC}$, 1361 peripheral venous catheters, 704 arterial catheters, and 395 pulmonary artery catheters) in hospitalized patients [30]. Chlorhexidine solutions were either an aqueous solution of $2 \%$ chlorhexidine ( 2 trials), a $70 \%$ alcoholic solution of $0.5 \%$ chlorhexidine ( 4 trials), an alcoholic solution of $1 \%$ chlorhexidine (1 trial), or a combination of $0.25 \%$ chlorhexidine, $0.025 \%$ benzalkonium chloride and $4 \%$ benzylic alcohol ( 1 trial). Catheter insertion sites and duration of catheterization were comparable between the two groups. The use of chlorhexidine rather than povidone iodine aqueous solution significantly reduced catheter-related bloodstream infections by approximately 50\% (RR: 0.51 [95\% CI, 0.27-0.97]). For every 1000 catheter sites disinfected with chlorhexidine solutions rather than povidone iodine solutions, 71 episodes of CVC colonization and 11 episodes of infections would be prevented. Similar findings with an alcoholic formulation of $2 \%$ chlorhexidine were reported after publication of the metaanalysis [31], confirming that aqueous povidone iodine should not be used for this indication.

In most of these studies, chlorhexidine's superiority was explained, at least in part, by a synergistic effect with alcohol, even for low chlorhexidine concentrations. This synergistic effect was also demonstrated with povidone iodine. A randomized multicenter crossover trial compared the effectiveness of two pre-insertion cutaneous antisepsis protocols using aqueous $10 \%$ povidone-iodine or a solution of $5 \%$ povidone iodine in $70 \%$ ethanol [32]. The incidences of catheter colonization (RR: 0.38 [95\% CI: 0.22-0.65]) and catheter-related infection (RR: 0.34 [95\% CI: 0.13-0.91]) were significantly lower in patients managed using the alcoholic povidone iodine solution protocol compared to the aqueous povidone iodine solution protocol. No significant effect was observed on bloodstream infections, but the study was underpowered to explore this issue.

Only one trial has compared a chlorhexidine-based solution to $5 \%$ alcoholic povidone iodine. A total of 538 catheters were randomized and 481 (89.4\%) produced evaluable culture results [33]. Compared to alcoholic povidone iodine, the used of a chlorhexidine-based solution significantly reduced the incidence of catheter colonization by $50 \%$ ( $11.6 \%$ vs $22.2 \% \mathrm{p}=0.002$; incidence density, 9.7 vs 18.3 per 1000 catheter-days). The use of the chlorhexidine-based solution was also associated with a trend toward lower rates of catheter-related bloodstream infection $(1.7 \%$ vs $4.2 \% \mathrm{p}=0.09$; incidence density, 1.4 vs 3.4 per 1000 catheter-days). In this study, independent risk factors for catheter colonization were catheter insertion in the jugular vein (RR: 2.01 [95\% CI: 1.24-3.24]) and use of alcoholic povidone iodine as skin disinfectant (RR: 1.87 [95 CI: 1.18-2.96]). Although more studies are needed to confirm these results, chlorhexidine-based solutions do seem to be more effective than povidone iodine, even in an alcoholic formulation, and should be used as first-line antiseptics for $\mathrm{CVC}$ care.

Tolerance to chlorhexidine-based solutions is generally excellent. Contact dermatitis is occasionally observed whatever the formulation used and severe anaphylactic reactions have been exceptionally reported (less than 100 cases in the world).

\section{Antibiotic prophylaxis}

No studies have demonstrated any reduction in CVC infection rates with oral or parenteral antibacterial or antifungal drugs given during catheter insertion. In contrast, numerous studies have reported that antibiotic administration in patients with a CVC in situ significantly reduced the risk of catheter colonization and of blood stream infections [24]. In pediatric patients, two studies have assessed vancomycin prophylaxis for CVC flushing (antibiotic lock); both demonstrated a significant reduction in catheter-related bloodstream infection without any effect on mortality [34,35]. Because prophylactic use of vancomycin is an independent risk factor for vancomycin-resistant Enterococcus (VRE) acquisition, the risk of VRE emergence likely outweighs the benefit of using prophylactic vancomycin. Systemic antibiotic 
prophylaxis should not be used during catheter insertion or maintenance just for the purpose of preventing catheter infection.

\section{Tunneling}

Subcutaneous tunneling of short-term CVCs is thought to reduce the incidence of catheter infection, presumably by increasing the distance between the venous entry site and skin emergence. Catheter emergence in a skin area that is less colonized by skin pathogens is another possible mechanism. Another advantage of tunneling is better fixation of the catheter. Evidence from studies on tunneling efficacy have suggested that this technique reduces CVC infections in patients with short-term devices, where most colonized pathogens arise from the catheter insertion site. A meta-analysis of randomized controlled trials demonstrated that tunneling decreased catheter colonization by $39 \%$ and bloodstream infection by $44 \%$ compared to non-tunneling [36]. These results were partly due to one trial with CVCs inserted via the internal jugular vein, and no significant risk reduction was observed when only the data from five subclavian catheter trials were pooled. Mechanical complications or difficulties during placement were not increased by tunneling but these outcomes were not evaluated in depth. Although, this meta-analysis concluded that tunneling decreased catheter-related infections, the data do not support routine subcutaneous tunneling of shortterm venous catheters unless subclavian access is not possible (or contraindicated) and the duration of catheterization is anticipated to be more than 7 days.

\section{Dressing}

Because occlusive dressings trap moisture on the skin and provide an ideal environment for quick local microflora growth, dressings for insertion sites must be permeable to water vapor. The two most common types of dressing used are sterile, transparent, semi-permeable polyurethane dressings coated with a layer of an acrylic adhesive, and gauze and tape dressings. Transparent, semipermeable polyurethane dressings have become a popular way of dressing catheter insertion sites because they allow continuous visual inspection of the site, allow patients to have baths and to shower without saturating the dressing, and require less frequent changes than do standard gauze and tape dressings; finally these dressings are time-saving for the staff. However, as there is no evidence regarding which type of dressing provides the greatest protection against infection the choice of dressing can be a matter of preference. If blood is oozing from the catheter insertion site, a gauze dressing may be preferred.

In a meta-analysis, the use of a chlorhexidine-impregnated sponge placed over the site of short-term vascular and epidural catheters significantly reduced the risk of catheter colonization but not catheter-related bloodstream infection compared to standard dressing [37]. More recently, a study performed in seven ICUs in France included 1636 patients randomized to receive catheter dressings with or without a chlorhexidine gluconate-impregnated sponge [38]. A total of 3778 catheters (28,931 catheter-days) were evaluated. The median duration of catheter insertion was 6 (interquartile range, 4-10) days. Use of chlorhexidine gluconateimpregnated sponge dressings decreased the rates of major catheter-related infections (10/1953 [0.5\%], 0.6 per 1000 catheter-days vs $19 / 1825$ [1.1\%], 1.4 per 1000 catheter-days; hazard ratio [HR], 0.39 [95\% CI, 0.17$0.93] ; \mathrm{p}=0.03)$ and catheter-related bloodstream infections $(6 / 1953$ catheters, 0.40 per 1000 catheter-days vs $17 / 1825$ catheters, 1.3 per 1000 catheter-days; HR, 0.24 [95\% CI, 0.09-0.65]). Use of chlorhexidine gluconateimpregnated sponge dressings was not associated with greater resistance of bacteria in skin samples at catheter removal and was well tolerated. The authors concluded that the use of chlorhexidine gluconate-impregnated sponge dressings with intravascular catheters in the ICU reduced the risk of infection even when background infection rates were low, and should be recommended [38]. However, the antiseptic solution used for catheter care was povidone iodine. As previously discussed, chlorhexidine is more effective than povidone iodine to disinfect the skin. Therefore, whether there is any benefit from using chlorhexidine-impregnated sponge for catheters in patients in whom chlorhexidine is used for catheter care remains unknown.

The optimal frequency for routine changing of catheter dressings is unknown. It is probably of little use to change dressing before 7 days, except when the insertion site is soiled with blood or moisture or the dressing is unstuck [38]. The dressing site should be disinfected with the same antiseptic solution used for catheter placement.

\section{Venous line maintenance}

The optimal time interval for routine replacement of intravenous administration sets has been studied in three well-controlled trials [39-41]. Replacing administration sets no more frequently than 72 hours after initiation of use is safe and cost-effective [42]. Because blood, blood products, and lipid emulsions (including parenteral nutrition and propofol) have been identified as independent risk factors for catheter-related infection [43], tubing used to administer these products should be replaced within 24 hours or immediately after the end of administration.

An aseptic technique is very important when accessing the system. Catheter, tubing, or syringe manipulations must be done only after cleaning hands with an alcoholbased handrub solution. Hubs and sampling ports should 
- Use protocols for catheter insertion and maintenance

- Check for adequate training, experience, and numbers of nurses caring for patients with CVC

- Use antimicrobial-coated CVCs if the incidence of catheter-related infection remains high despite adherence to guidelines and recommended measures

- Use maximal sterile-barrier precautions during catheter insertion

- Insert catheters using the subclavian venous site

- $\quad$ Use ultrasound guidance during catheterization (?)

- Consider tunneling if subclavian access is not possible and the CVC is anticipated to be in situ for more than 7 days

- Clean hands with an alcohol-based handrub solution before any manipulation of the infusion line

- Change dressings not more frequently than 7 days if not soiled, wet, or unstuck

- Avoid the use of antibiotic prophylaxis at catheter insertion, and antibiotic ointments or inline filters during catheter maintenance

- Use the enteral route or peripheral venous access instead of the CVC as soon as possible

- Do not schedule routine catheter changes

- $\quad$ Remove catheters when they are no longer needed

be disinfected with chlorhexidine-based antiseptic solutions before accessing [44]. During prolonged catheterization, infection risk is strongly connected to the duration of catheter stay and frequent catheter hub access increases catheter-related infection risk from colonized catheter hubs rather than from the insertion site. The number of manipulations of the central venous line, especially when an aseptic technique is not respected, increases the risk of catheter-related bloodstream infection. The use of the enteral or oral route to deliver drugs and diet should, thus, be encouraged whenever possible.

The continued need for the catheter should be assessed every day and removal considered when the catheter is no longer essential for medical management. Catheter replacement at scheduled time intervals as a method to reduce catheter-related infection has not been shown to be beneficial $[45,46]$. Scheduled guidewire exchanges of catheters have also been proposed, but a meta-analysis of 12 randomized controlled trials failed to demonstrate any reduction in infection rates with routine guidewire exchange compared to catheter replacement on an asneeded basis [47]. On the contrary, exchanging catheters with the use of a guidewire increases the risk of bloodstream infection, while replacement involving insertion of catheters at new sites increases the risk of mechanical complications [46]. Thus, routine replacement of CVCs is not necessary for functional catheters with no evidence of local or systemic complications. Catheter guidewire exchange is acceptable for replacement of a nonfunctional catheter.

Application of antibiotic or antiseptic ointments (e.g., bacitracin, mupirocin, neomycin, and polymyxin) to catheter-insertion sites increases the rate of catheter colonization by fungi, promotes the emergence of antibiotic-resistant bacteria, and has not been shown to lower the rate of catheter-related bloodstream infections [48]. These ointments should not be used. No data are available to support the efficacy of in-line filters in preventing infections associated with intravascular catheters and infusion systems, although the use of these devices increases the cost of the venous line. Administration of prophylactic heparin reduces the risk of thrombosis around the catheter. Because thrombi and fibrin deposits on catheters may be a nidus for microbial colonization of intravascular catheters, anticoagulant therapy may have a role in prevention [49]. Moreover, these agents are also indicated in the management of inbed patients with multiple risk factors for venous thrombosis.

\section{Conclusion}

Catheter-related bloodstream infection remains the most serious complication of central venous access and a leading cause of nosocomial infection in the ICU. Prevention of catheter-related infection involves several measures which should be used in combination (Table 1) [50-52]. The most important include the use of a checklist to guide catheter insertion and maintenance; adequate training of the nursing staff involved in the management of vascular access and an adequate patientto-nurse ratio; the use of maximal sterile barrier precautions during catheter insertion; preference for a chlorhexidine-based solution for skin antisepsis and use of the subclavian vein whenever possible; cleaning hands with an alcohol-based handrub solution before any manipulation of the infusion line; and removing any useless catheters. The use of antimicrobial-coated CVCs should be reserved for ICUs where the incidence of catheter-related infection remains high despite adherence 
to guidelines and recommended measures. As with any device used in the ICU, healthcare workers caring for a patient with a central venous access device need to be adequately trained, and assessed as being competent in using $\mathrm{CVCs}$ and adhering to infection prevention practices.

\section{Abbreviations}

$\mathrm{Cl}=$ confidence interval, $\mathrm{CVC}=$ central venous catheter, $\mathrm{HR}=$ hazard ratio, $\mathrm{ICU}=$ intensive care unit, $R R=$ relative risk, $V R E=$ vancomycin-resistant Enterococcus.

\section{Competing interests}

$\mathrm{OM}$ has received research and educational grants as well as consulting fees from: 3M, Cardinal Health, Bayer Healthcare and VIATRIS. CD-F and DF declare that they have no competing interests.

\section{Published: 9 March 2010}

\section{References}

1. Mermel LA: Prevention of intravascular catheter-related infections. Ann Intern Med 2000, 132:391-402.

2. O'Grady NP, Alexander M, Dellinger EP, et al.: Guidelines for the prevention of intravascular catheter-related infections. Infect Control Hosp Epidemiol 2002, 23:759-769

3. Herrmann M, Lai QJ, Albrecht RM, Mosher DF: Adhesion of Staphylococcus aureus to surface-bound platelets: role of fibrinogen/fibrin and platelet integrins. J Infect Dis 2003, 167:312-322.

4. Centers for Disease Control and Prevention (2001) Issues in healthcare settings: CDC's 7 Healthcare Safety Challenges. Available at: http://www.cdc gov/ncidod/dhqp/about_challenges.html. Accessed November 26, 2009

5. Berenholtz SM, Pronovost PJ, Lipsett PA, et al.: Eliminating catheter-related bloodstream infections in the intensive care unit. Crit Care Med 2004, 32:2014-2020.

6. Warren, DK, Zack JE, Mayfield JL, et al:: The effect of an education programme on the incidence of central venous catheter-associated bloodstream infection in a medical ICU. Chest 2004, 126:1612-1618.

7. Eggimann P, Harbarth S, Constantin MN, Touveneau S, Chevrolet JC, Pittet D: Impact of a prevention strategy targeted at vascular-access care on incidence of infections acquired in intensive care. Lancet 2000, 355:1864-1868.

8. L'Hériteau F, Olivier M, Maugat S: Impact of a five-year surveillance of central venous catheter infections in the REACAT intensive care unit network in France. J Hosp Infect 2007, 66:123-129.

9. Yilmaz G, Koksal I, Aydin K, Caylan R, Sucu N, Aksoy F: Risk factors of catheterrelated bloodstream infections in parenteral nutrition catheterization. J Parenter Enteral Nutr 2007, 31:284-287.

10. Zingg W, Imhof A, Maggiorini M, Stocker R, Keller E, Ruef C: Impact of a prevention strategy targeting hand hygiene and catheter care on the incidence of catheter-related bloodstream infections. Crit Care Med 2009, 37:2167-2173.

11. Barsuk JH, Cohen ER, Feinglass J, McGaghie WC, Wayne DB: Use of simulation-based education to reduce catheter-related bloodstream infections. Arch Intern Med 2009, 169:1420-1423.

12. Soifer NE, Borzak S, Edlin BR, Weinstein RA: Prevention of peripheral venous catheter complications with an intravenous therapy team: a randomized controlled trial. Arch Intern Med 1998, 158:473-477.

13. Puntis JW, Holden CE, Smallman S, Finkel Y, George RH, Booth IW: Staff training: a key factor in reducing intravascular catheter sepsis. Arch Dis Child 1991, 66:335-337.

14. Fridkin SK, Pear SM, Williamson TH, Galgiani JN, Jarvis WR: The role of understaffing in central venous catheter-associated bloodstream infections. Infect Control Hosp Epidemiol 1993, 17:150-158.

15. Alonso-Echanove J, Edwards JR, Richards MJ, et al.: Effect of nurse staffing and antimicrobial-impregnated central venous catheters on the risk for bloodstream infections in intensive care units. Infect Control Hosp Epidemiol 2003, 24:916-925

16. Maki DG, Ringer M: Risk factors for infusion-related phlebitis with small peripheral venous catheters: a randomized controlled trial. Ann Intern Med 1991, 114:845-854.
17. Sheth NK, Franson TR, Rose HD, Buckmire FL, Cooper JA, Sohnle PG: Colonization of bacteria on polyvinyl chloride and Teflon intravascular catheters in hospitalized patients. J Clin Microbio/ 1983, 18:1061-1063.

18. Halton KA, Cook DA, Whitby M, Paterson DL, Graves N: Cost effectiveness of antimicrobial catheters in the intensive care unit: addressing uncertainty in the decision. Crit Care 2009, 13:R35.

19. Raad I, Buzaid A, Rhyne J: Minocycline and ethylenediaminetetraacetate for the prevention of recurrent vascular catheter infections. Clin Infect Dis 1997, 25:149-151.

20. Ramritu P, Halton K, Collignon P: A systematic review comparing the relative effectiveness of antimicrobial-coated catheters in intensive care units. Am J Infect Control 2008, 36:104-117.

21. Kalfon P, de Vaumas C, Samba D: Comparison of silver-impregnated with standard multi-lumen central venous catheters in critically ill patients. Crit Care Med 2007, 35:1032-1039.

22. Hockenhull JC, Dwan KM, Smith GW: The clinical effectiveness of central venous catheters treated with anti-infective agents in preventing catheter-related bloodstream infections: a systematic review. Crit Care Med 2009, 37:702-712.

23. Dezfulian C, Lavelle J, Nallamothu BK, Kaufman SR, Saint S: Rates of infection for single-lumen versus multilumen central venous catheters: a metaanalysis. Crit Care Med 2003, 31:2385-2390.

24. Merrer J, De Jonghe B, Golliot F; French Catheter Study Group in Intensive Care: Complications of femoral and subclavian venous catheterization in critically ill patients: a randomized controlled trial. JAMA 2001, 286:700-707.

25. Ruesch $S$, Walder $B$, Tramèr MR: Complications of central venous catheters: internal jugular versus subclavian access - a systematic review. Crit Care Med 2002, 30:454-460.

26. Parienti JJ, Thirion M, Mégarbane B, et al:: Femoral vs jugular venous catheterization and risk of nosocomial events in adults requiring acute renal replacement therapy: a randomized controlled trial. JAMA 2008, 99:2413-2422

27. Randolph AG, Cook DJ, Gonzales CA, Pribble CG: Ultrasound guidance for placement of central venous catheters: a meta-analysis of the literature. Crit Care Med 1996, 24:2053-2058

28. Karakitsos D, Labropoulos N, De Groot E: Real-time ultrasound-guided catheterisation of the internal jugular vein: a prospective comparison with the landmark technique in critical care patients. Crit Care 10:R162.

29. Raad II, Hohn DC, Gilbreath BJ, et al.: Prevention of central venous catheterrelated infections by using maximal sterile barrier precautions during insertion. Infect Control Hosp Epidemiol 15:231-238.

30. Chaiyakunapruk N, Veenstra DL, Lipsky BA, Saint S: Chlorhexidine compared with povidone iodine solution for vascular catheter-site care: a metaanalysis. Ann Intern Med 2002, 136:792-801.

31. Balamongkhon B, Thamlikitkul V: Implementation of chlorhexidine gluconate for central venous catheter site care at Siriraj Hospital, Bangkok, Thailand. Am J Infect Control 2007, 35:585-588.

32. Parienti JJ, du Cheyron D, Ramakers M, et al: Alcoholic povidone-iodine to prevent central venous catheter colonization: a randomized unitcrossover study. Crit Care Med 2004, 32:708-713.

33. Mimoz O, Villeminey S, Ragot S: Chlorhexidine-based antiseptic solution versus alcohol-based povidone-iodine for central venous catheter care. Arch Intern Med 2007, 167:2066-2072.

34. Spafford PS, Sinkin RA, Cox C: Prevention of central venous catheter-related coagulase-negative staphylococcal sepsis in neonates. J Pediatr 1994, 125:259-263.

35. Kacica MA, Horgan MJ, Ochoa L, Sandler R, Lepow ML, Venezia RA: Prevention of gram-positive sepsis in neonates weighing less than $1500 \mathrm{~g}$ J Pediatr 1994, 125:253-258.

36. Randolph AG, Cook DJ, Gonzales CA, Brun-Buisson C: Tunneling short-term central venous catheters to prevent catheter-related infection: a metaanalysis of randomized, controlled trials. Crit Care Med 1998, 26:1452-1457.

37. Ho KM, Litton E: Use of chlorhexidine-impregnated dressing to prevent vascular and epidural catheter colonization and infection: a meta-analysis. J Antimicrob Chemother 2006, 58:281-287.

38. Timsit JF, Schwebel C, Bouadma L, et al:: Chlorhexidine-impregnated sponges and less frequent dressing changes for prevention of catheterrelated infections in critically ill adults: a randomized controlled trial. JAMA 2009, 301:1231-1241.

39. Maki DG, Botticelli JT, LeRoy ML, Thielke TS: Prospective study of replacing 
administration sets for intravenous therapy at 48-vs 72 -hour intervals: 72 hours is safe and cost-effective. JAMA 1987, 258:1777-1781.

40. Raad I, Hanna HA, Awad A, et al:: Optimal frequency of changing intravenous administration sets: is it safe to prolong use beyond 72 hours? Infect Control Hosp Epidemiol 2001, 22:136-139.

41. Band JD, Maki DG: Safety of changing intravenous delivery systems at longer than 24-hour intervals. Ann Intern Med 1979, 91:173-178.

42. Gillies D, O'Riordan L, Wallen M, Rankin K, Morrison A, Nagy S: Timing of intravenous administration set changes: a systematic review. Infect Control Hosp Epidemiol 2004, 25:240-250.

43. Sitges-Serra A, Linares J, Perez JL, Jaurrieta E, Lorente L: A randomized trial on the effect of tubing changes on hub colonization and catheter sepsis during parenteral nutrition. JPEN J Parenter Enteral Nutr 1985, 9:322-325.

44. Salzman MB, Isenberg HD, Rubin LG: Use of disinfectants to reduce microbial contamination of hubs of vascular catheters. J Clin Microbiol 1993, 31:475-479

45. Khalifa R, Dahyot-Fizelier C, Laksiri L: Indwelling time and risk of colonization of peripheral arterial catheters in critically ill patients. Intensive Care Med 2008, 34:1820-1826.

46. Cobb DK, High KP, Sawyer RG: A controlled trial of scheduled replacement of central venous and pulmonary artery catheter. N Engl J Med 1992, 327:1062-1068

47. Cook D, Randolph A, Kemerman P, et al:: Central venous catheter replacement strategies: A systematic review of the literature. Crit Care Med 1997, 25:1417-1424.

48. Zakrzewska-Bode A, Muytjens HL, Liem KD, Hoogkamp-Korstanje JA: Mupirocin resistance in coagulase-negative staphylococci, after topical prophylaxis for the reduction of colonization of central venous catheters. J Hosp Infect 1995, 31:189-193.

49. Randolph AG, Cook DJ, Gonzales CA, Andrew M: Benefit of heparin in central venous and pulmonary artery catheters: a meta-analysis of randomized controlled trials. Chest 1998, 113:165-171.

50. Pronovost P, Needham D, Berenholtz S: An intervention to decrease catheter-related bloodstream infections in the ICU. N Engl J Med 2006, 355:2725-2732.

51. Timsit JF: Diagnosis and prevention of catheter-related infections. Curr Opin Crit Care 2007, 13:563-571.

52. Mermel LA, Farr BM, Sherertz RJ et al:: Guidelines for the management of intravascular catheter-related infections. Infect Control Hosp Epidemiol 2001 22:222-242.

doi:10.1186/cc8853

Cite this article as: Frasca D, et al: Prevention of central venous catheterrelated infection in the intensive care unit. Critical Care 2010, 14:212. 